\title{
Bacterial Product Template Domain: An Evolutionary Intermediate between Dehydratase and Aldol Cyclase of Type I Polyketide Synthases
}

\section{yuanyuan Feng}

Shanghai Jiao Tong University

\section{Xu Yang}

Shanghai Jiao Tong University

Huining Ji

Shanghai Jiao Tong University

\section{Zixin Deng}

Wuhan University https://orcid.org/0000-0003-0724-3390

Shuangjun Lin

Shanghai Jiao Tong University

Jianting Zheng ( $\boldsymbol{\nabla}$ jtzheng@sjtu.edu.cn )

Shanghai Jiao Tong University https://orcid.org/0000-0003-1250-3556

\section{Article}

Keywords: Bacterial, Dehydratase, Aldol Cyclase, Polyketide Synthases

Posted Date: December 6th, 2021

DOI: https://doi.org/10.21203/rs.3.rs-1069407/v1

License: (c) (1) This work is licensed under a Creative Commons Attribution 4.0 International License. Read Full License

Version of Record: A version of this preprint was published at Communications Biology on May 26th, 2022. See the published version at https://doi.org/10.1038/s42003-022-03477-8. 


\section{Abstract}

The product template (PT) domains act as an aldol cyclase to control the regiospecific aldol cyclization of the extremely reactive poly- $\beta$-ketone intermediate assembled by an iterative type I polyketide synthases (PKSs). Up to now, only the structure of fungal PksA PT that mediates the first-ring cyclization via C4-C9 aldol cyclization is available. We describe here the structural and computational characterization of a bacteria PT domain that controls C2-C7 cyclization in orsellinic acid (OSA) synthesis. Mutating the catalytic His949 of the PT abolishes production of OSA and results in a tetraacetic acid lactone (TTL) generated by spontaneous $\mathrm{O}-\mathrm{C}$ cyclization of the acyl carrier protein (ACP)-bound tetraketide intermediate. Crystal structure of the bacterial PT domain closely resembles dehydrase (DH) domains of modular type I PKSs in the overall fold, dimerization interface and catalytic "His-Asp" dyad organization, but is significantly different from PTs of fungal iterative type I PKSs. QM/MM calculation reveals that the catalytic His949 abstracts a proton from C2 and transfers it to C7 carbonyl to mediate the cyclization reaction. According to the structural similarity to DHs and the functional similarity to fungal PTs, we propose that the bacterial PT represents an evolutionary intermediate between the two tailoring domains of type I PKSs.

\section{Introduction}

Polyketides are structurally diverse natural products, constituting one of the most important sources of bioderived pharmaceuticals, for example, antibiotics, antitumor agents, and immunosuppressants [1, 2]. They are biosynthesized from coenzyme A (CoA)-activated carboxylic acids by multifunctional megaenzymes or mutisubunit enzyme complexes called polyketide synthases (PKSs) that resemble fatty acid synthases (FASs), using a $\beta$-ketoacyl synthase (KS), acyl transferase (AT) and acyl carrier protein (ACP). The resulting ACP-bound poly- $\beta$-keto thioester intermediates are ultimately converted to highly diversified compounds by optional tailoring domains. PKSs are grouped into different types according to their biosynthetic machinery architectures, including modular type I PKSs, iterative type I PKSs, iterative type II PKS, and the ACP-independent freestanding iterative type III PKSs. Modular type I PKSs are widely distributed in bacteria, comprising multiple sets of domains that are organized into modules. In contrast, the iterative type I PKSs are usually found in fungal species, repeatedly reusing one module that is highly programmed to generate polyketides with diverse structural features by using different domain combinations in each iteration.

Iterative type I PKSs are further classified as non-reducing (NR), partial-reducing (PR), and highly-reducing (HR) PKSs according to the function and phylogeny[3-5]. NR-PKSs synthesize aromatic polyketides that have important biological activities, exemplified by the environmental carcinogen aflatoxin B1. The poly$\beta$-ketone backbone assembled by a NR-PKS is extremely reactive. Spontaneous cyclization must be suppressed to form the unique cyclization pattern observed in the final product. PksA involved in biosynthesis of aflatoxin B1 is a model system to investigate NR-PKSs. Functional dissection of PksA reveals a unique product template (PT) domain between AT and ACP domains, which functions as an aldol cyclase mediating the regioselective cyclization of a hexanoyl-primed octaketide intermediate via 
C4-C9/C2-C11 aldol condensations to generate a key intermediate of aflatoxin biosynthesis, norsolorinic acid (Figure 1) [6, 7].

NR-PKSs are divided into three basal subclades (subclades I-III) according to a phylogenetic analysis of KS sequences [8]. PTs embedded in subclades I and II are classified into five group based on a phylogenetic analysis of those associated with known aromatic polyketides [9]. The well-studied PksA PT and other NR-PKS PTs involved in C4-C9/C2-C11 cyclization belong to Group IV. PTs of group I perform the regioselective $\mathrm{C} 2-\mathrm{C} 7$ cyclization of a tetraketide primed by different starter units and are embedded in NR-PKSs that synthesize polyketides containing a single aromatic ring, exemplified by the orsellinic acid synthase (OSAS) from Aspergillus nidulans [10, 11]. PTs of group II catalyze the C2-C7 cyclization of pentaketide intermediates to form the first aromatic ring of tetrahydroxynaphthalene (THN) compounds, of which the second ring is formed by thioesterase-catalyzed Claisen cyclization [12]. PTs of group III and group V mediate cyclization of longer intermediates to form polyketides containing multiply fused-ring structures. Group III PTs have C2-C7 regioselectivity while group V PTs cyclize the nascent polyketide via C6-C11/C4-C13 regioselectivity [9]. NR-PKSs in subclade III contain an additional methyltransferase (CMeT) domain compared to those in subclade I and II and produce aromatics with $\mathrm{C} 2-\mathrm{C} 7$ cyclization regioselectivity. The PTs of subclade III NR-PKSs are classified into groups VI and VII [13]. Recently, phylogenetic analysis of all known fungal NR-PKSs reveals a new group VIII PT domains [14].

Only the structure of PksA PT has been reported, displaying a distinct double hotdog (DHD) fold, a variant of that observed in the dehydrase (DH) domains of modular type I PKSs [7, 15]. PksA PT is dimeric in crystal structures, indicating the role of PT domains in dimerization of fungal NR-PKSs. A PT-specific sequence insertion is involved in dimerization of the two monomers and conserved in all known fungal NR-PKSs. Each monomer contains an internal pocket that extends $30 \AA$ from the surface to the bottom for substrate binding and is visually divided into the phosphopantetheine (PPANT)-binding region, the cyclization chamber, and the hydrophobic hexyl-binding region. The PT active site is a His-Asp "catalytic dyad" located at the cyclization chamber. The His residue functions as a catalytic base, using its Nס as the basic nitrogen to deprotonate the substrate, while the Asp polarizes the His by hydrogen bond to $N \varepsilon$. A binary structure complexed with a bisisoxazole model of the experimentally inaccessible poly- $\beta$-ketone intermediate reveals additional protein-substrate interactions involved in orientation and stabilization during the multistep catalytic cycle. As the only PT domain that has been structurally characterized, PksAPT provides a vital model for understanding the high regioselective aldol cyclization of poly- $\beta$-ketone intermediates assembled by NR-PKSs.

Orsellinic acid (OSA) is synthesized in bacteria and, in most case, functions as a building block that is further incorporated into biosynthesis of more complex natural products, for example, avilamycin produced by Streptomyces viridochromogenes [16] and calicheamicin produced by Micromonospora echinospora [17]. Cloning and analysis of biosynthetic gene clusters suggests that AviM and CalO5 are responsible for the assembly of OSA. Cultures of Streptomyces lividans TK24 and Streptomyces coelicolor $\mathrm{CH} 999$ containing AviM can produce OSA, confirming the function of AviM as OSAS [16]. Compared to fungal OSASs [11], AviM and CalO5 lack the starter-unit acyltransferase (SAT) domain 
loading a primer for polyketide extension and the thioesterase (TE) domain releasing OSA from the PPANT group of the ACP domain (Figure 1A). Here, we confirm that AviM PT domain functions as an aldol cyclase to catalyze $\mathrm{C2}-\mathrm{C} 7$ cyclization in OSA synthesis by comparing the products generated by AviM and its mutants. A tetraacetic acid lactone (TTL, 1$)$ is generated by spontaneous $0-C$ cyclization of the ACP-bound tetraketide backbone when the catalytic His949 of the PT domain is replaced by Ala or Phe. The structure of AviM PT, solved by selenomethionine incorporation and single-wavelength anomalous dispersion (SAD) phasing, closely resembles dehydratase (DHs) of modular type I PKSs in the overall fold, dimerization interface and catalytic "His-Asp" dyad organization. The catalytic His949 of AviM PT uses its $N \varepsilon$ as the basic nitrogen to deprotonate the substrate, whereas the catalytic His1345 of PksA PT uses its Nס as the basic nitrogen $[7,15]$. QM/MM calculation confirms that the catalytic His949 of AviM PT abstracts a proton from $\mathrm{C} 2$ and transfers it to $\mathrm{C} 7$ carbonyl in the aldol cyclization reaction. The structural similarity to DHs and the functional similarity to PTs suggest that the bacterial PT may represent an evolutionary intermediate between the two tailoring domains of type I PKSs.

\section{Methods}

\section{General Methods and Materials}

Strain cultivation and DNA manipulation followed standard methods. DNA sequencing and all primers were purchased from Tsingke Biotechnology Co., Ltd. (China). Primers used for PCR and site-directed mutagenesis are listed in Table S1. All Fast Digest enzymes were from Thermo Scientific. DNA polymerases were purchased from Invitrogen Trading Co., Ltd. (China). All the other chemicals and reagents were purchased from commercial suppliers and used without further purification. ClustalX was used in protein sequence alignments.

\section{Cloning and Site-directed Mutagenesis}

aviM was cloned from S. viridochromogenes Tü57 and digested with Ndel and Hindll restriction enzymes. The $3.9 \mathrm{~kb}$ DNA fragment was purified and ligated into pET28a to generate pET28-AviM. aviM PT was cloned and digested with $N d e l$ and $E c o R I$ restriction enzymes to obtain an 816 bp gene fragment, which was purified and ligated into pET28a to generate pET28-aviM-PT. The sfp gene was amplified from the genomic DNA of Bacillus subtilis. All constructs contain $\mathrm{His}_{6} \mathrm{~N}$-terminal tags for purification.

Site-directed mutations were generated by PCR using standard protocol according to the GeneTailor method (Invitrogen). Template plasmid was removed by Dpnl digestion at $37^{\circ} \mathrm{C}$ for 1.5 hours. All constructed plasmids were confirmed by DNA sequencing.

\section{Protein Expression and Purification}

The E. coli BL21(DE3) cells transformed with corresponding plasmids were grown to an $\mathrm{OD}_{600}$ of 0.4 in LB medium supplemented with $50 \mathrm{mg} / \mathrm{mL}$ kanamycin at $37^{\circ} \mathrm{C}$ and induced with $0.3 \mathrm{mM}$ IPTG for $12 \mathrm{~h}$ at 
$16^{\circ} \mathrm{C}$. The cells were harvested by centrifugation and resuspended in lysis buffer containing $500 \mathrm{mM}$ $\mathrm{NaCl}, 50 \mathrm{mM}$ Tris ( $\mathrm{pH}$ 7.5). Following sonication, cell debris was removed by centrifugation at $15,000 \mathrm{~g}$ for 40 minutes. The supernatant was poured over a column of Nickel-NTA resin, which was then washed with $50 \mathrm{~mL}$ lysis buffer containing $30 \mathrm{mM}$ imidazole and eluted with lysis buffer containing $300 \mathrm{mM}$ imidazole. Proteins were further purified by using a Superdex 200/Superose 6 gel filtration column (GE Healthcare Life Sciences) equilibrated with buffer containing $150 \mathrm{mM} \mathrm{NaCl}$ and $10 \mathrm{mM}$ Tris (pH 7.5). All purification steps were carried out at $4{ }^{\circ} \mathrm{C}$.

\section{Heterologous Production of OSA}

E. coli K207-3 was transformed with pET28a and its AviM derivates. E. coli K207-3 containing pET-28a does not lead to any OSA production and served as a negative control. The corresponding overnight preculture was used for inoculation of $50 \mathrm{~mL}$ of LB medium. $1 \mathrm{mM}$ isopropyl- $\beta$-D-1-thiogalactopyranoside (IPTG) was supplemented at OD600 of 0.4 . After $24 \mathrm{~h}$ of incubation at $30^{\circ} \mathrm{C}$ and $220 \mathrm{rpm}$, the culture was acidified with concentrated $\mathrm{HCl}$ to $\mathrm{pH} 4$ and extracted with ethyl acetate $(\mathrm{v} / \mathrm{v}, 1: 1)$. The organic layer was dried and dissolved in $0.5 \mathrm{~mL}$ of methanol. LC-MS was conducted with an Agilent 1290 Infinity Liquid chromatography and 6545 Quadrupole Time-of-Flight Mass Spectrometer by using negative electrospray ionization and a C18 reverse-phase column (TC-C18(2) $4.6 \times 250 \mathrm{~mm}, 5 \mu \mathrm{m}$, Agilent). The flow rate was $0.4 \mathrm{~mL} / \mathrm{min}$, and a binary solvent system gradient of $0.1 \%$ formic acid in water (A) and in acetonitrile (B) was used as follows: 0-10 min: 20-40\% B, 10-20 min: 40\% B, 20-30 min: 40-50\% B, 30-32 min: 50-100\% B, 32-35 min: $100 \%$ B, 35-36 min: 100-20\% B, 36-45 min: 20\% B. For MS/MS, HPLC system was coupled to a 6545XT AdvanceBio LC/Q-TOF mass spectrometer (Agilent Technologies) equipped with an ESI interface (Agilent Technologies) operating in negative ion mode using a capillary voltage of $+4 \mathrm{kV}$. Other parameters were drying gas temperature, $325^{\circ} \mathrm{C}$; drying gas flow, $13 \mathrm{~L} / \mathrm{min}$; and nebulizing gas pressure, 2 bar. Detection was carried out within a mass range of $50-1100 \mathrm{~m} / \mathrm{z}$. The MS/MS analyses were acquired by precursor ion scan: m/z 167.035, RT: $12.5 \mathrm{~min}$, dwell time: $1 \mathrm{~min}$. CE at $2 \mathrm{ev}, 10 \mathrm{ev}, 20 \mathrm{ev}$ and $40 \mathrm{ev}$.

\section{In vitro Enzymatic Assay of AviM}

For the enzymatic assays of AviM, a typical $100 \mu \mathrm{l}$ reaction mixture contains $10 \mathrm{mM} \mathrm{MgCl}, 0.2 \mathrm{mM}$ CoA, $50 \mu \mathrm{M}$ WT AviM or its mutant and $10 \mu \mathrm{M}$ sfp in $10 \mathrm{mM}$ Tris, $\mathrm{pH} 7.4,150 \mathrm{mM} \mathrm{NaCl}$, and $10 \%$ glycerol. After incubation at $30^{\circ} \mathrm{C}$ for $1 \mathrm{~h}$, the reaction mixture was supplemented with $1 \mu \mathrm{l}$ of acetyl-CoA $(100 \mathrm{mM})$ and 2 $\mu \mathrm{l}$ of malonyl-CoA $(100 \mathrm{mM})$ and incubated at $25^{\circ} \mathrm{C}$. The reaction was quenched with $5 \mu \mathrm{l}$ of $6 \mathrm{mM} \mathrm{HCl}$ and the solution was vortexed to precipitate the enzyme. Then the mixture was spun at $14,800 \mathrm{rpm}$ for 10 min and the supernatant was subjected to LC-MS analysis. The method for LC-MS is as described as in vivo analysis.

\section{TTL Synthesis}

The synthesis of TTL has been described [18]. 2,2,6-Trimethyl-4H-1,3-dioxin-4-one 1 (3 ml, $22.5 \mathrm{mmol}, 1.0$ eq) and $1 \mathrm{H}$-Benzotriazole $2(1.0 \mathrm{eq})$ in dry $\mathrm{PhMe}(80 \mathrm{~mL})$ were heated for $11 \mathrm{~h}$ at $90^{\circ} \mathrm{C}$. Solvents were removed by rotary evaporator and a pale yellow solid 3 was generated. 1 (3.0 eq) was added dropwise to 
$\mathrm{LiN}\left(\mathrm{SiMe}_{3}\right)_{2}$ (3.1eq) in THF $(750 \mathrm{ml})$ at $-78^{\circ} \mathrm{C}$ and stirred for $1.5 \mathrm{~h}$. Then, 3 was added and the mixture was warmed up to room temperature and stirred overnight. The reaction mixture was quenched with $1 \mathrm{M}$ aqueous $\mathrm{HCl}$ to $\mathrm{pH} 4$. The mixture was extracted with EtOAc $(3 \times 250 \mathrm{ml})$, followed by washing with brine and drying with $\mathrm{MgSO}_{4}$. The solvent was removed by rotary evaporator and the residual was dissolved in $\mathrm{CH}_{2} \mathrm{Cl}_{2}(250 \mathrm{ml})$ and washed with the Sigma-Aldrich 456101 buffer solution (pH 9.0, $\left.5 \times 50 \mathrm{ml}\right)$. The organic layer was dried with $\mathrm{MgSO}_{4}$ and the solvents were removed by rotary evaporator. The crude product was purified by silica gel column chromatography with hexanes/EtOAc (8:1 to 2:1) to give diketo1,3-dioxinone 4 as a clear oil. 4 (100 mg, $0.44 \mathrm{mmol})$ in PhMe $(30 \mathrm{ml})$ was heated for $8 \mathrm{~h}$ at $110{ }^{\circ} \mathrm{C}$. The solvents were removed by rotary evaporator and the products were separated by silica gel column chromatography (hexanes:EtOAc=2:1) to provide TTL as a yellowish oil. TTL: ${ }^{1} \mathrm{H} N M R(400 \mathrm{MHz}$, DMSOd6): $\delta$ (ppm) 2.17 (s, 3H), 3.75 (s, 2H), $5.27(\mathrm{~d}, J=4 \mathrm{~Hz}, 1 \mathrm{H}), 6.05(\mathrm{~d}, J=4 \mathrm{~Hz}, 1 \mathrm{H}) ;{ }^{13} \mathrm{C}$ NMR $(100 \mathrm{MHz}$, DMSO-d6): $\delta$ (ppm) 30.23, 47.73, 89.38, 103.08, 160.35, 164.20, 170.66, 203.31.

\section{Phylogenetic Analysis}

A dataset of 36 proteins was selected, including 27 PTs (2 from bacteria (AviM PT, Cal05 PT), 25 from fungi) with a supplement of a Branching domain from rhizoxin PKS as the outgroup[19]. The sequences were aligned with MAFFT-linsi (v7.480)[20] and trimmed with trimAL (v1.2)[21]. The final alignments have 279 trimmed columns. A phylogenetic tree was constructed by IQ-TREE (v1.6.12)[22] with "LG+G4" model and 1000 ultrafast bootstraps. The phylogenetic tree is edited by iTol.

\section{Crystallization and Structure Determination}

Crystallization of the Avim PT was performed using $1 \mu \mathrm{l}$ of protein soulution $(8 \mathrm{mg} / \mathrm{mL}$ in $150 \mathrm{mM}$ sodium chloride, $10 \mathrm{mM}$ Tris-HCl pH 7.5) mixed with $1 \mu \mathrm{l}$ of reservoir solution by sitting drop vapor diffusion at $20^{\circ} \mathrm{C}$. The best crystals were obtained in a solution containing $0.1 \mathrm{M} \mathrm{Tris-HCl}(\mathrm{pH} 8.5), 1 \mathrm{M}$ lithium chloride, 28\% PEG 4000 (w/v) within 5 days. Selenomethionyl crystals were obtained in a similar condition. The selenomethionyl and native data were collected at wavelengths of $0.97853 \AA$ at $100 \mathrm{~K}$. All the datasets were collected at the beamline BL-18U1, Shanghai Synchrondron Radiation Facility (SSRF), China, and processed using HKL2000. Crystals belonged to space group $\mathrm{P} 2{ }_{1}$ with similar unit-cell dimensions for the native and selenomethionyl crystals. Experimental phases were determined using AutoSol in Phenix. The model was iteratively built in Coot[23] and refined in Refmac[24].

\section{Computational Details}

The tetraketide-PPANT substrate was docked into the active site of AviM PT by AutoDock software[25]. The parameters for tetraketide-PPANT were generated at the HF/6-31G(d) level with the Gaussian 16 program[26]. The restrained electrostatic potential (RESP)[27] charge fitting procedure was employed on the tetraketide-PPANT and the missing parameters were generated by the Antechamber package. The complex was solvated in a cubic box of TIP3P water molecules with $10 \AA$ thickness and $\mathrm{Na}^{+}$counterions were added to achieve charge neutralization. MD simulations of the complex were run with the AMBER 
software package (version 18)[28] . The enzymes and substrate were described under ff12SB and GAFF force fields from the AMBER18 software package. The Particle Mesh Ewald (PME) [29] method was conducted to account for long-range electrostatic interactions. The SHAKE algorithm was employed to fix angles and bonds involving hydrogen atoms[30]. Prior to production MD simulations, the solvated system was treated with minimization and progressive heating, with the system temperature increasing from 0 to $298 \mathrm{~K}$ over 50 ps. After 50 ps equilibration, the complex conducted six-times 50 ns production simulations with a different random number without any restraints under NPT conditions. Then, the conformation that favored the reaction taking place was selected as the initial structure for the $Q M / M M$ calculations. The QM calculations were performed with the M06-2x/6-31G(d) method and the MM region was under the AMBER Parm99 force field, utilizing a two-layered ONIOM method in the Gaussian 16 program. We selected the side chain of the H949, D1104 as well as a portion of the backbone of A959 and V1123. We also selected the entire tetraketide, the sulphur atom and the two adjacent $\mathrm{CH} 2$ groups of the substrate as QM region. Additionally, the $\mathrm{H}_{2} \mathrm{O}$ that was positioned between the substrate $\mathrm{C} 7$ carbonyl and the D1104 side chain was retained at the QM layer. The rest of the system was treated as the MM region.

\section{Results}

\section{AviM PT Catalyzes C2-C7 Cyclization of OSA}

AviM is previously shown to synthesize OSA in S. lividans TK24 and S. coelicolor CH999 [16]. Here we used E. coli K207-3 [31], an E. coli B-derived strain developed for the heterologous expression of polyketide biosynthetic genes, to investigate the function of AviM in vivo. The DNA fragments encoding AviM was amplified by PCR and ligated into pET28a. The resulting pET28a-aviM was transformed into $E$. coli K207-3 to give the recombinant strain for OSA production, while the E. coli K207-3 carrying the vector $\mathrm{pET} 28 \mathrm{a}$ was used as the control strain. LC-MS analysis revealed that cultures of $E$. coli K207-3 containing pET28a-aviM were able to produce OSA (Figure 2).

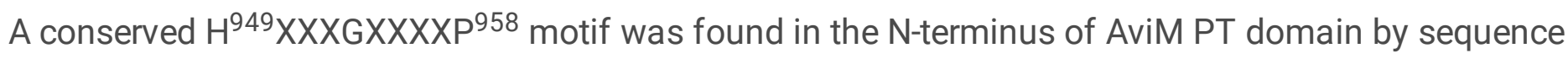
alignment of AviM with various fungal NR-PKSs (Figure S1). The His949 residue, corresponding the catalytic His1345 of PksA PT, was replaced by site-specific mutagenesis with Ala or Phe. LC-MS analysis showed that the mutations of His949Ala and His949Phe completely abolished OSA production of AviM in E. coli K207-3. However, the extracted ion chromatography (EIC) revealed a new product that had the same molecular weight but different retention time relative to OSA. MetFrag was used to identified the new product with a relative mass deviation of $5 \mathrm{ppm}$ and an absolute mass deviation of $0.002 \mathrm{Da}$ as thresholds [32]. Tetraacetic acid lactone (TTL, 1) was retrieved from PubChem as a potential candidate (Figure S2). Subsequently, the new product was identified as TTL by comparing with the standard prepared by organic synthesis (Figure 2A). Obviously, the functions of the KS, AT, and ACP domains are not affected by mutations in the PT domain. The new TTL product is generated by spontaneous O-C cyclization of the ACP-bound tetraketide backbone in the absence of PT domain (Figure 1B). In contrast, a $\mathrm{C}-\mathrm{C}$ bond is formed between $\mathrm{C} 2$ and $\mathrm{C} 7$ by aldol cyclization in the presence of PT domain, followed by 
aromatization, to yield final OSA product. Analysis of the LC-MS data of the wild type AviM also revealed the presence of TTL, whereas the production of TTL was significantly increased in H949A and H949F mutants (Figure 2A). Interestingly, a triacetic acid lactone (TAL, 2) released after two condensation steps was also observed in the cultures of the wild-type AviM and mutating the catalytic H949 also increased the production of the TAL (Figure S3)

In vitro functional assays of AviM were carried out to exclude the possible effects of other enzymes encoded in E. coli K207-3 genome. The recombinant AviM was expressed in E. coli BAP1 containing a sfp gene from Bacillus subtilis [33]. The purified AviM was further incubated with coenzyme A (CoA) and sfp enzyme to ensure the phosphopantetheinylation of all proteins. Trace amount of OSA was indeed produced when AviM was incubated with acetyl-CoA and malonyl-CoA in vitro (Figure 2B). These in vitro and in vivo results confirm that AviM alone can mediate the assembly and release of OSA. Replacing the putative catalytic His949 by the Ala residue destroyed the aldol cyclase activity of AviM PT domain. TTL instead of OSA was produced by the AviM His949Ala mutant. In short, these in vivo and in vitro assays establish that the PT domain of AviM functions as an aldol cyclase to control the C2-C7 cyclization in OSA biosynthesis. Consequently, we proposed a OSA biosynthesis pathway presented in (Figure 1B). The poly- $\beta$-keto chain elongation first starts with an acetyl-CoA building block. After two iterative rounds of decarboxylative Claisen condensations using malonyl building blocks, some intermediates are released by spontaneous $\mathrm{O}-\mathrm{C}$ cyclization to yield a shunt product TAL. The remaining intermediates are condensed with the third malonyl-CoA molecule to form the ACP-bound tetraketide intermediate which were cyclized between $\mathrm{C} 2$ and $\mathrm{C} 7$ by the PT domain and released as OSA.

\section{Overall Structure of AviM PT Resembles DHs instead of PksA PT}

PT domains catalyze the first-ring cyclization via three common patterns ( $22-\mathrm{C} 7, \mathrm{C} 4-\mathrm{C} 9$, and $\mathrm{C} 6-\mathrm{C} 11)$. Up to now, only PksA PT (group IV) that cyclize the first ring via C4-C9 regiospecificity has been structurally characterized, hindering attempts to rationally control the cyclization patterns of poly- $\beta$-keto backbones. Our in vivo and in vitro functional assays revealed that AviM PT controls the C2-C7 cyclization in OSA biosynthesis. To understand the molecular basis of the aldol cyclization step, we solved the crystal structure of the AviM PT domain.

Diffraction-quality crystals were obtained by sitting-drop method using polyethylene glycol 4000 as a precipitant. The protein crystallized in space group $\mathrm{P} 2_{1}$ with two independent monomers per asymmetric unit. The structure was solved to $2.0-\AA$ A resolution through selenomethionine incorporation and SAD phasing (Table S2). The histidine-tag and the first ten residues are invisible in both monomers. Residues 946-950 and 1055-1069 are invisible in monomer B but visible in monomer $A$. The two monomers in the asymmetric unit have essentially identical folds with a rmsd of $0.4 \AA$ for Ca positions (Figure S4). An automatic search of AviM PT for structurally homologous proteins using the Dali server revealed low sequence identity (less than 20\%) to all previously reported structures. Interestingly, AviM PT shows more structural similarity to dehydratase (DH) domains than to PksA PT. Each monomer comprises a DHD fold of DH domains (Figure 3), characterized by a continuous antiparallel $\beta$-sheet curving around two a-helices 
(aHD1 from the N-terminal and aHD2 from the C-terminal hotdog folds). The C-terminal hotdog (residues 1056-1174) possesses an additional helix (aC) comparted to the N-terminal hotdog (residues 915-1032). These two hotdogs are connected by a 23-residue-long flexible linker containing two short helices (aL1 and aL2). AviM PT most closely resembles the DH domain from CurF module of curacin PKS (Protein Data Bank (PDB) codes 3KG6, $2.4 \AA$ Ca rmsd; 19\% sequence identity) [34]. The most notable difference is in the C-terminal hotdog of CurF DH, which contains an additional $3_{10}$ helix (Figure S4). The PksA PT is 70-residue larger than the AviM PT. They are superimposed with a rmsd of $3.2 \AA$ for 147 (of 260 possible) Ca positions. Compared to AviM PT, four obvious differences are observed in PksA PT (Figure 3B): 1) the N-terminal hotdog and the $\mathrm{C}$-terminal hotdog are connected by a bent long helix; 2) the aHD1 from the $\mathrm{N}$-terminal hotdog is two turns longer; 3 ) the $\beta N 1$ and $\beta N 2$ strands of the $\mathrm{N}$-terminal hotdog are much longer; and 4) the ten C-terminal residues of the PksA PT form a helix that cap the substrate binding tunnel.

The two monomers in the asymmetric unit dimerize through $\mathrm{N}$-terminal $\beta$ strands and bury a surface area of $665 \AA^{2}$ (calculated by PDBe PISA). The relatively large dimer interface and the observed migration behavior as a dimer on the size exclusion column suggest that the PT domain is involved in oligomerization of AviM (Figure S5). The $\beta$-hairpin turns between $\beta N 1$ and $\beta N 2$, and between $\beta N 5$ and $\beta N 6$ contribute significantly to the dimer interface (Figure 3A). Deleting the PT domain of AviM (residues 915-1174) abolished the production of both OSA and TTL (Figure 2A), suggesting that the deletion of PT probably caused an architectural change rendering the megaenzyme inactive. The angle between the two monomers is $\sim 180^{\circ}$ in CurF DH and this extended conformation has been observed in all previously reported structures of PKS DH domains. However, the two monomers of AviM PT are obviously in a different relative orientation, forming an angle of $\sim 150^{\circ}$ (Figure S4C and D). The observed conformation of the excised PT domain may reflect the conformation in the complete AviM megacomplex. Interestingly, the dimer interface of AviM PT is completely different from that observed in PksA PT, of which the two monomers dimerize through the $\mathrm{C}$-terminal hotdog, involving the $\mathrm{aC}$ helix, the $\beta$ strand following the bent long helix linker, and the linker connecting aHD2 and $\beta 10$ (Figure 3A).

\section{AviM PT Possesses a Substrate Tunnel Distinct from DHs and PksA PT}

The "catalytic dyad" of AviM PT is in the similar orientation as observed in DH domains. The His949 is from the "cap loop" of the N-terminal hotdog and the Asp1104 from the helix aHD2 of the C-terminal hotdog. The Nס of His949 is involved in a hydrogen bond with the backbone carbonyl oxygen of lle956, indicating that the catalytic His949 using uses its $\mathrm{N} \varepsilon$ as the basic nitrogen to deprotonate the substrate (Figure 4). The Asp1104 is $5 \AA$ from the His 949 and the Asp1104Ala mutant shows decreased production of OSA (Figure 2A), indicating that the Asp1104 may be involved in catalysis indirectly. In contrast, the catalytic Asp 1543 directly polarizes the catalytic His1345 by a hydrogen bond to $N \varepsilon$ in PksA PT and mutating Asp1543 results in no detectable activity (Figure 4x) $[7,15]$. The active site is positioned at the middle of a $25 \AA$ substrate binding tunnel that is located on top of two DHD helices and closed off by the cap loop (Figure 4B). The substrate binding tunnel starts at the junction of the $\mathrm{N}$ - and $\mathrm{C}$-terminal hotdog and extends into the $\mathrm{C}$-terminal hotdog fold. The entrance is bounded by the $\beta \mathrm{N} 3 \mathrm{~b}$, the $\beta \mathrm{C} 3$, and the $\mathrm{C}$ - 
terminal end following the $\beta \mathrm{C} 6$, while the bottom is bounded by the $\mathrm{C}$-terminal of the $\mathrm{aC}$ and the $\mathrm{N}$ terminal of the $\beta C 1$. The volume of the AviM PT substrate binding tunnel $\left(761 \AA^{3}\right)$ is as same as that of CurF DH $\left(762 \AA^{3}\right)$, but is significantly smaller than that of PksA PT $\left(1196 \AA^{3}\right)$, which contains an additional hydrophobic binding region lying at the bottom of the tunnel to accommodate the hexyl group derived from the hexanoyl-CoA starter unit. Compared to the tunnel of PksA PT that is closed off at the bottom by the $\beta$ strands of the C-terminal hotdog (Figure 4C), the tunnels of AviM PT and CurF DH are open at the bottoms (Figure 4B and 4D). Interestingly, the bottom of the tunnel is differently positioned in AviM PT and CurF DH due to the alternative orientation of the $\mathrm{aC}$ helix. The bottom is positioned on top of the middle of the aHD2 in AviM PT. However, in CurF DH, the bottom is positioned at different side of $\mathrm{aC}$ helix and located on top of the C-terminal end of the aHD2. As a result, the tunnel of AviM PT is straight while the tunnel of CurF DH is bent almost $90^{\circ}$ at the catalytic His 1720.

\section{QM/MM Calculation of C2-C7 Cyclization Catalyzed by AviM PT}

The proposed mechanism for AviM PT-catalyzed cyclization involves formation of an enolate intermediate by deprotonation of $\mathrm{C} 2$ and subsequent aldol addition to the $\mathrm{C} 7$ carbonyl (Scheme 1). Electrostatic potential surface indicates the substrate tunnel is negatively charged (Figure S6). The polar microenvironment favors the $\mathrm{C} 7$ carbonyl in its electrophilic keto tautomeric form. We used QM/MM calculation to understand the molecular basis of the $\mathrm{C} 2-\mathrm{C} 7$ cyclization. The structure of PksA PT complexed with the bisisoxazole guided the placement of the tetraketide-PPANT substrate in the active site of AviM PT [15]. The resulting complex was subjected to MD simulations. The "active" conformer was selected according to two distances $(\mathrm{d}(\mathrm{N} \varepsilon-\mathrm{Ha})$ and $\mathrm{d}(\mathrm{C} 2-\mathrm{C} 7))$ and optimized at the ONIOM(M062X/6-31G(d): Amber) level. In the resulting reactant, the $\mathrm{C} 1$ and $\mathrm{C} 3$ carbonyl of the substrate form hydrogen bonds with the backbone $\mathrm{NH}$ of $\mathrm{A} 959$ and $\mathrm{V} 1123$ respectively. In addition, a water molecule is positioned between the substrate $\mathrm{C} 7$ carbonyl and the D1104 side chain (Figure 5). In the first stage, the $\mathrm{C} 2$ is deprotonated by the $\mathrm{N} \varepsilon$ of the catalytic H949. The first transition state (TS1) was observed when the $\mathrm{H}$ was located at $1.53 \AA$ from the $\mathrm{C} 2$ and at $1.23 \AA$ from the $\mathrm{N} \varepsilon$ (Figure $5 \mathrm{~B}$ ). The $\mathrm{C} 1-\mathrm{C} 2$ and $\mathrm{C} 2-\mathrm{C} 3$ bonds decrease from $1.51 \AA$ in reactant to $1.40 \AA$ in the first intermediate (IM1), while the $\mathrm{C}=0$ bonds of C1 and C3 increase from $1.21 \AA$ in reactant to $1.23 \AA$, suggesting the formation of an enolate structure. Consequently, the negative charge is distributed between the $\mathrm{C} 2$ and two adjacent carbonyls. Next, the nucleophilic $\mathrm{C} 2$ launches an attack on the C7. Simultaneously, the $\mathrm{C} 7$ carbonyl deprotonates the $\mathrm{H} 949 \mathrm{~N} \varepsilon$ to form a hydroxy. During the process, the $\mathrm{H}$ of the $\mathrm{H} 949 \mathrm{~N} \varepsilon$ gradually turns to the $\mathrm{C} 7$ carbonyl accompanying the decrease of the distance between $\mathrm{C} 2$ and $\mathrm{C} 7$. In TS2, the $\mathrm{d}(\mathrm{C} 2-\mathrm{C} 7)$ and $\mathrm{d}(\mathrm{H}-\mathrm{O} 7)$ distances are $2.56 \AA$ and $1.15 \AA$ respectively, whereas they are further decreased to $1.56 \AA$ and $0.99 \AA$ in IM2. The deprotonation of $\mathrm{C} 2$ conquers a $14.0 \mathrm{kcal} / \mathrm{mol}$ energy barrier and the subsequent ring cyclization overcomes a $14.1 \mathrm{kcal} / \mathrm{mol}$ barrier (Figure 5C and Figure S9). The two steps are feasible with low reaction barriers. Besides, the deprotonation and aldol addition are exergonic with $7.5 \mathrm{kcal} / \mathrm{mol}$. Following aromatization could occur with a loss of hydroxide by an E1cb stepwise mechanism as proposed for PksA PT [7]. 


\section{Discussion}

NR-PKSs utilize PT domains to mediate the regioselective cyclization of highly reactive poly- $\beta$-ketone intermediates. According to the phylogenetic analysis, fungal PTs are divided into 8 groups. OSAS PTs belong to the group I and perform the regioselective C2-C7 cyclization. Compare to their fungal counterparts, bacterial OSASs lack the SAT and TE domains. Our in vitro assays indicate that the AviM alone can synthesize OSA from acetyl-CoA and malonyl-CoA, but is inefficient (Figure 2B). It's possible that other enzymes help the initiation and/or release steps in vivo. AviM PT has low sequence similarity to fungal PTs. We confirmed the aldol cyclase of AviM PT by mutating its catalytic His949. The TTL derived from spontaneous $\mathrm{O}-\mathrm{C}$ cyclization of the ACP-bound tetraketide was produced both in vivo and in vitro.

The overall structure and the active site of AviM PT resemble DHs of modular type I PKSs instead of the PksA PT. Compared to AviM PT, PksA PT uses different structural elements to form the dimer interface and has an additional helix to cap the substrate binding tunnel. The His-Asp "catalytic dyad" is also oriented differently. In PksA PT, the side chain of the catalytic Asp1543 makes a hydrogen bond with the $\mathrm{N} \varepsilon$ of the catalytic His1345 [7]. Therefore, the polarized His1345 use its Nס as the basic nitrogen to deprotonate the substrate. In contrast, the catalytic His949 of AviM PT is polarized by a neighboring backbone carbonyl oxygen and use its $\mathrm{N} \varepsilon$ as the basic nitrogen, as observed in DHs of modular type I PKSs. The corresponding Asp1104 is $5 \AA$ from the His949 and the Asp1104Ala mutant still retains more $10 \%$ catalytic activity (Figure $2 \mathrm{~A}$ ). QM/MM calculations demonstrate that the His949 initiates the reactions by abstracting a proton from $\mathrm{C} 2$ and transfers it to $\mathrm{C} 7$ carbonyl during formation of the $\mathrm{C} 2-\mathrm{C} 7$ bond (Figure 5). We built a model of CalO5 PT, another bacterial OSAS PT, by using AlphaFold [35]. The structure is similar to AviM PT both in overall structure and in organization of His-Asp "catalytic dyad" (Figure S7). Models of four fungal OSAS PTs were also built and showed overall similarity to PksA PT. Iterative type I PKSs are usually found in fungi, whereas Wang et al recently discoverers numerous iterative type I PKSs in Streptomyces and proposes the horizontal-gene-transfer hypothesis on the origin of fungal iterative type I PKSs [36]. The observation, that AviM PT structurally resembles DHs of modular type I PKSs but catalytically acts as an aldol cyclase, suggests that bacterial PTs may be evolutionary intermediates between two tailoring domains of type I PKSs. The phylogenetic tree constructed by PTs and DHs shows that all the sequences are consistent with host species. Bacterial PTs nest within the clade containing DHs. These two groups of enzymes make up a large clade that is sister to fungal PTs. Bacterial PTs are phylogenetically distant from the fungal PTs but close to DHs, consistent with the functional and structural analysis (Figure S8).

In summary, AviM PT acts as an aldol cyclase, mediating C2-C7 cyclization in OSA biosynthesis. It's inactivation results in generation of TTL derived from spontaneous O-C cyclization of the ACP-bound tetraketide. Structural comparison reveals that the dimer interface, the overall architecture, and the "catalytic dyad" of AviM PT are similar to DHs of modular type I PKSs. These results suggest that bacterial PTs may represent an evolutionary intermediate between DHs and PTs of type I PKSs. 


\section{Declarations}

\section{Data Availability}

Atomic coordinates of two structures have been deposited in PDB with accession codes 7VWK.

\section{Supplementary Data}

Supplementary Data are available Online.

\section{Acknowledgements}

This work was supported by National Key R\&D Program of China (2020YFA0907900 and 2019YFA0905400) and National Natural Science Foundation of China $(32070040,31770068,31800045)$. We thank Prof. Qianjin Kang for helpful discussion about the TTL identification and the Shanghai Synchrotron Radiation Facility beamlines BL18U1 for diffraction data collection.

\section{Conflicts of Interest}

The authors declare that they have no conflicts of interest.

\section{References}

1. Klaus, M. and M. Grininger, Engineering strategies for rational polyketide synthase design. Nat Prod Rep, 2018. 35(10): p. 1070-1081.

2. Robbins, T., et al., Structure and mechanism of assembly line polyketide synthases. Curr Opin Struct Biol, 2016. 41: p. 10-18.

3. Vederas, J.C., Explorations of fungal biosynthesis of reduced polyketides - a personal viewpoint. Nat Prod Rep, 2014. 31(10): p. 1253-9.

4. Townsend, C.A., Aflatoxin and deconstruction of type l, iterative polyketide synthase function. Nat Prod Rep, 2014. 31(10): p. 1260-5.

5. Simpson, T.J., Fungal polyketide biosynthesis - a personal perspective. Nat Prod Rep, 2014. 31(10): p. $1247-52$.

6. Crawford, J.M., et al., Deconstruction of iterative multidomain polyketide synthase function. Science, 2008. 320(5873): p. 243-6.

7. Crawford, J.M., et al., Structural basis for biosynthetic programming of fungal aromatic polyketide cyclization. Nature, 2009. 461(7267): p. 1139-43. 
8. Kroken, S., et al., Phylogenomic analysis of type I polyketide synthase genes in pathogenic and saprobic ascomycetes. Proc Natl Acad Sci U S A, 2003. 100(26): p. 15670-5.

9. $\mathrm{Li}, \mathrm{Y} ., \mathrm{W} . \mathrm{Xu}$, and $\mathrm{Y}$. Tang, Classification, prediction, and verification of the regioselectivity of fungal polyketide synthase product template domains. J Biol Chem, 2010. 285(30): p. 22764-73.

10. Bok, J.W., et al., Chromatin-level regulation of biosynthetic gene clusters. Nat Chem Biol, 2009. 5(7): p. 462-4.

11. Schroeckh, V., et al., Intimate bacterial-fungal interaction triggers biosynthesis of archetypal polyketides in Aspergillus nidulans. Proc Natl Acad Sci U S A, 2009. 106(34): p. 14558-63.

12. Fujii, I., et al., Enzymatic synthesis of 1,3,6,8-tetrahydroxynaphthalene solely from malonyl coenzyme A by a fungal iterative type I polyketide synthase PKS1. Biochemistry, 2000. 39(30): p. 8853-8.

13. Ahuja, M., et al., Illuminating the diversity of aromatic polyketide synthases in Aspergillus nidulans. J Am Chem Soc, 2012. 134(19): p. 8212-21.

14. Liu, L., et al., Bioinformatical analysis of the sequences, structures and functions of fungal polyketide synthase product template domains. Sci Rep, 2015. 5: p. 10463.

15. Barajas, J.F., et al., Polyketide mimetics yield structural and mechanistic insights into product template domain function in nonreducing polyketide synthases. Proc Natl Acad Sci U S A, 2017. 114(21): p. E4142-E4148.

16. Gaisser, S., et al., Cloning of an avilamycin biosynthetic gene cluster from Streptomyces viridochromogenes Tu57. J Bacteriol, 1997. 179(20): p. 6271-8.

17. Ahlert, J., et al., The calicheamicin gene cluster and its iterative type / enediyne PKS. Science, 2002. 297(5584): p. 1173-6.

18. Basseta, J.-F., et al., Studies on the resorcylates: biomimetic total syntheses of (+)-montagnetol and (+)-erythrin. Tetrahedron Letters, 2010. 51(5): p. 783-785.

19. Bretschneider, T., et al., Vinylogous chain branching catalysed by a dedicated polyketide synthase module. Nature, 2013. 502(7469): p. 124-8.

20. Katoh, K., et al., MAFFT: a novel method for rapid multiple sequence alignment based on fast Fourier transform. Nucleic Acids Res, 2002. 30(14): p. 3059-66.

21. Capella-Gutiérrez, S., J.M. Silla-Martínez, and T. Gabaldón, trimAl: a tool for automated alignment trimming in large-scale phylogenetic analyses. Bioinformatics, 2009. 25(15): p. 1972-3.

22. Nguyen, L.T., et al., IQ-TREE: a fast and effective stochastic algorithm for estimating maximumlikelihood phylogenies. Mol Biol Evol, 2015. 32(1): p. 268-74. 
23. Emsley, P. and K. Cowtan, Coot: model-building tools for molecular graphics. Acta Crystallogr D Biol Crystallogr, 2004. 60(Pt 12 Pt 1): p. 2126-32.

24. Vagin, A.A., et al., REFMAC5 dictionary: organization of prior chemical knowledge and guidelines for its use. Acta Crystallogr D Biol Crystallogr, 2004. 60(Pt 12 Pt 1): p. 2184-95.

25. Morris, G.M., et al., AutoDock4 and AutoDockTools4: Automated docking with selective receptor flexibility. Journal of computational chemistry, 2009. 30(16): p. 2785-2791.

26. M. J. Frisch, G.W.T., H. B. Schlegel, G. E. Scuseria, M. A. Robb, J. R. Cheeseman, G. Scalmani, V. Barone, G. A. Petersson, H. Nakatsuji, X. Li, M. Caricato, A. V. Marenich, J. Bloino, B. G. Janesko, R. Gomperts, B. Mennucci, H. P. Hratchian, J. V. Ortiz, A. F. Izmaylov, J. L. Sonnenberg, D. Williams-Young, F. Ding, F. Lipparini, F. Egidi, J. Goings, B. Peng, A. Petrone, T. Henderson, D. Ranasinghe, V. G. Zakrzewski, J. Gao, N. Rega, G. Zheng, W. Liang, M. Hada, M. Ehara, K. Toyota, R. Fukuda, J. Hasegawa, M. Ishida, T. Nakajima, Y. Honda, O. Kitao, H. Nakai, T. Vreven, K. Throssell, J. A. Montgomery, Jr., J. E. Peralta, F. Ogliaro, M. J. Bearpark, J. J. Heyd, E. N. Brothers, K. N. Kudin, V. N. Staroverov, T. A. Keith, R. Kobayashi, J. Normand, K. Raghavachari, A. P. Rendell, J. C. Burant, S. S. Iyengar, J. Tomasi, M. Cossi, J. M. Millam, M. Klene, C. Adamo, R. Cammi, J. W. Ochterski, R. L. Martin, K. Morokuma, O. Farkas, J. B. Foresman, and D. J. Fox. 2016, Gaussian, Inc.: Wallingford CT.

27. Cornell, W.D., et al., A Second Generation Force Field for the Simulation of Proteins, Nucleic Acids, and Organic Molecules. J. Am. Chem. Soc, 1995. 117: p. 5179-5197.

28. Case, D.A., et al., D.A. Case, et al., Editors. 2018: University of California, San Francisco. p. amber.

29. Darden, T., D. York, and L. Pedersen, Particle Mesh Ewald: An N $\%$ og $(N)$ Method for Ewald Sums in Large Systems. J Chem Phys, 1993. 98: p. 10089-10092.

30. Ryckaert, J.-P., G. Ciccotti, and H.J.C. Berendsen, Numerical integration of the cartesian equations of motion of a system with constraints: molecular dynamics of n-alkanes. Journal of Computational Physics, 1977. 23(3): p. 327-341.

31. Murli, S., et al., Metabolic engineering of Escherichia coli for improved 6-deoxyerythronolide B production. J Ind Microbiol Biotechnol, 2003. 30(8): p. 500-9.

32. Ruttkies, C., et al., MetFrag relaunched: incorporating strategies beyond in silico fragmentation. J Cheminform, 2016. 8: p. 3.

33. Wu, J., et al., Construction and performance of heterologous polyketide-producing K-12-and Bderived Escherichia coli. Lett Appl Microbiol, 2010. 51(2): p. 196-204.

34. Akey, D.L., et al., Crystal structures of dehydratase domains from the curacin polyketide biosynthetic pathway. Structure, 2010. 18(1): p. 94-105. 
35. Jumper, J., et al., Highly accurate protein structure prediction with AlphaFold. Nature, 2021. 596(7873): p. 583-589.

36. Wang, B., et al., Unraveling the iterative type I polyketide synthases hidden in Streptomyces. Proc Natl Acad Sci U S A, 2020. 117(15): p. 8449-8454.

\section{Figures}


A $\begin{gathered}\text { Bacterial } \\ \text { AviM/CalO5 }\end{gathered}$

\section{KS}

AT PT

\section{Fungal OAS/TerA}

B
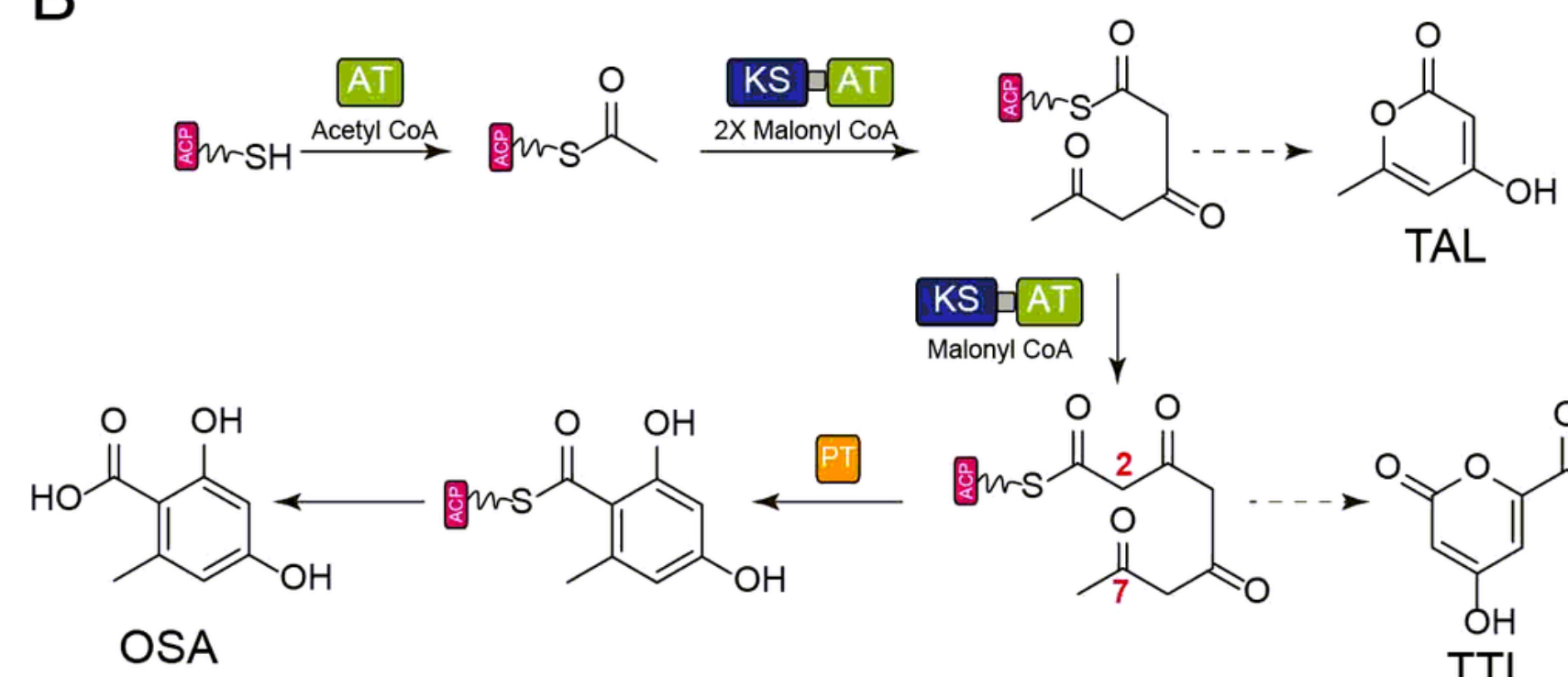

$\underset{\text { Malonyl CoA }}{\text { KS }}$

TAL

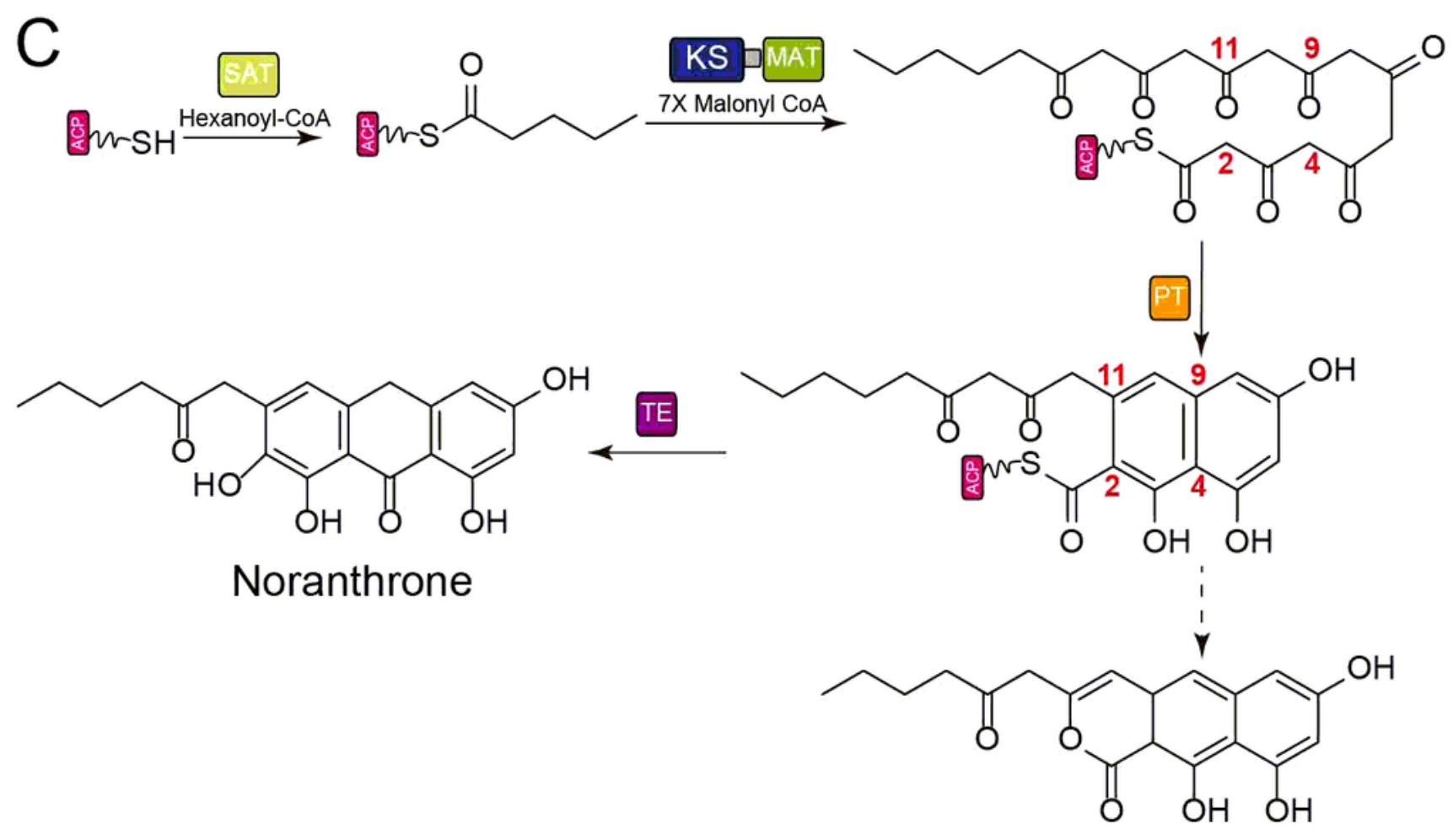

Figure 1

Biosynthesis reaction of OSA and Aflatoxin. (A) The domain compositions of bacterial and fungal OSASs. Bacterial OSASs lack the starter-unit acyltransferase (SAT) and the thioesterase (TE) domains. (B) Biosynthesis of OSA in bacteria. Triacetic acid lactone (TAL) released after two condensation steps and tetraacetic acid lactone (TTL) are generated in the absence of PT due to the spontaneous $0-C$ 
cyclization. AviM PT catalyzes the C2-C7 cyclization. (C) Biosynthesis reaction of Aflatoxin. PksA PT catalyzes the C4-C9 and C2-C11 cyclization.

A

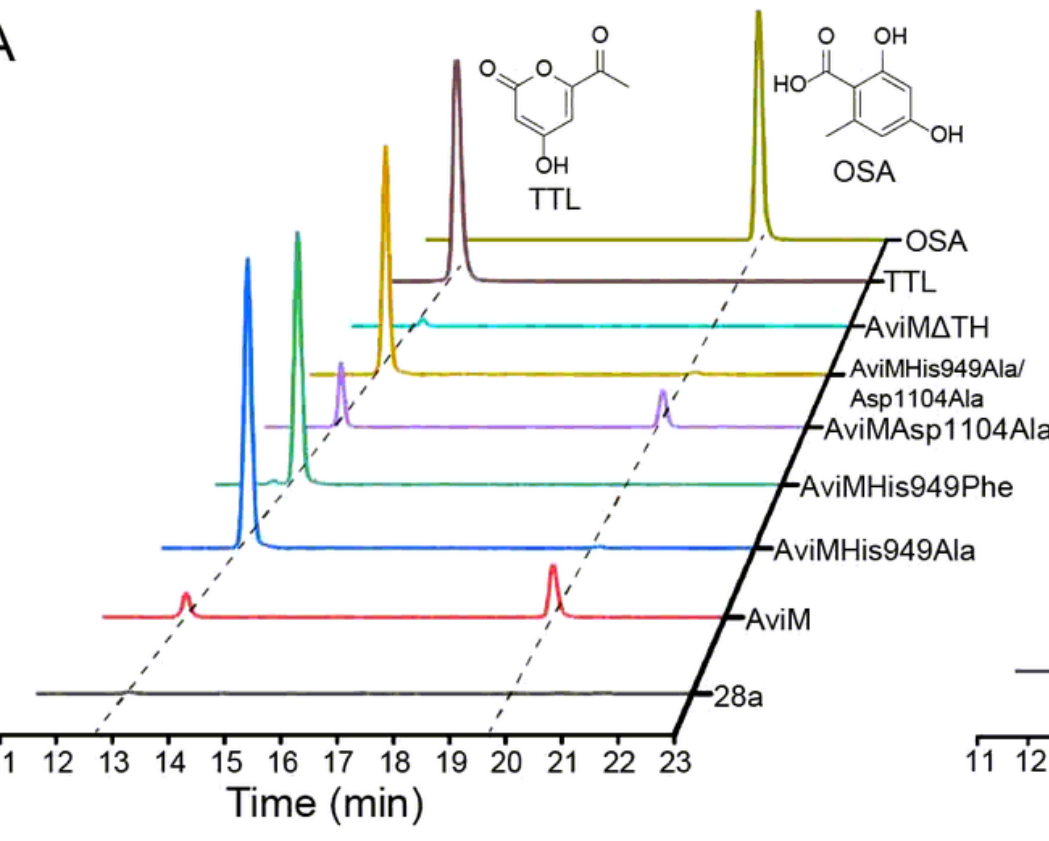

B

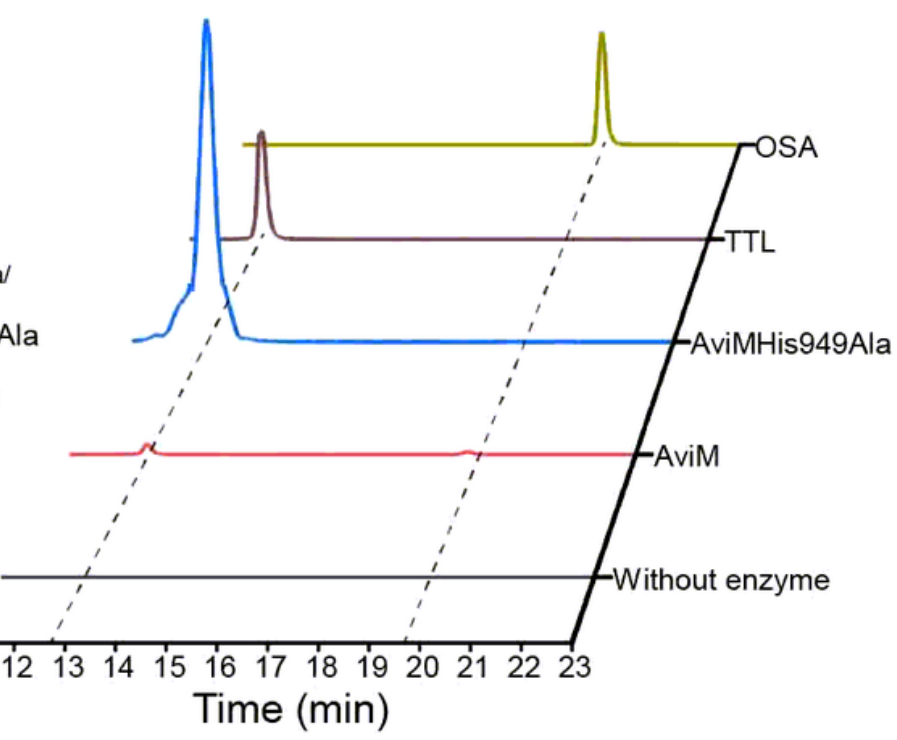

Figure 2

In vivo and in vitro assays of AviM-catalyzed OSA biosynthesis. (A) E. coli K207-3 expressing AviM synthesizes OSA. Mutating the catalytic $\mathrm{H} 949$ abolishes OSA production, but results in TTL from spontaneous O-C cyclization of the ACP-bound tetraketide backbone. (B) OSA is produced when AviM is incubated with acetyl-CoA and malonyl-CoA in vitro, while TTL is produced by AviM mutant. The traces show the extracted ion counts (m/z: 167.0350) monitored in the negative mode. 

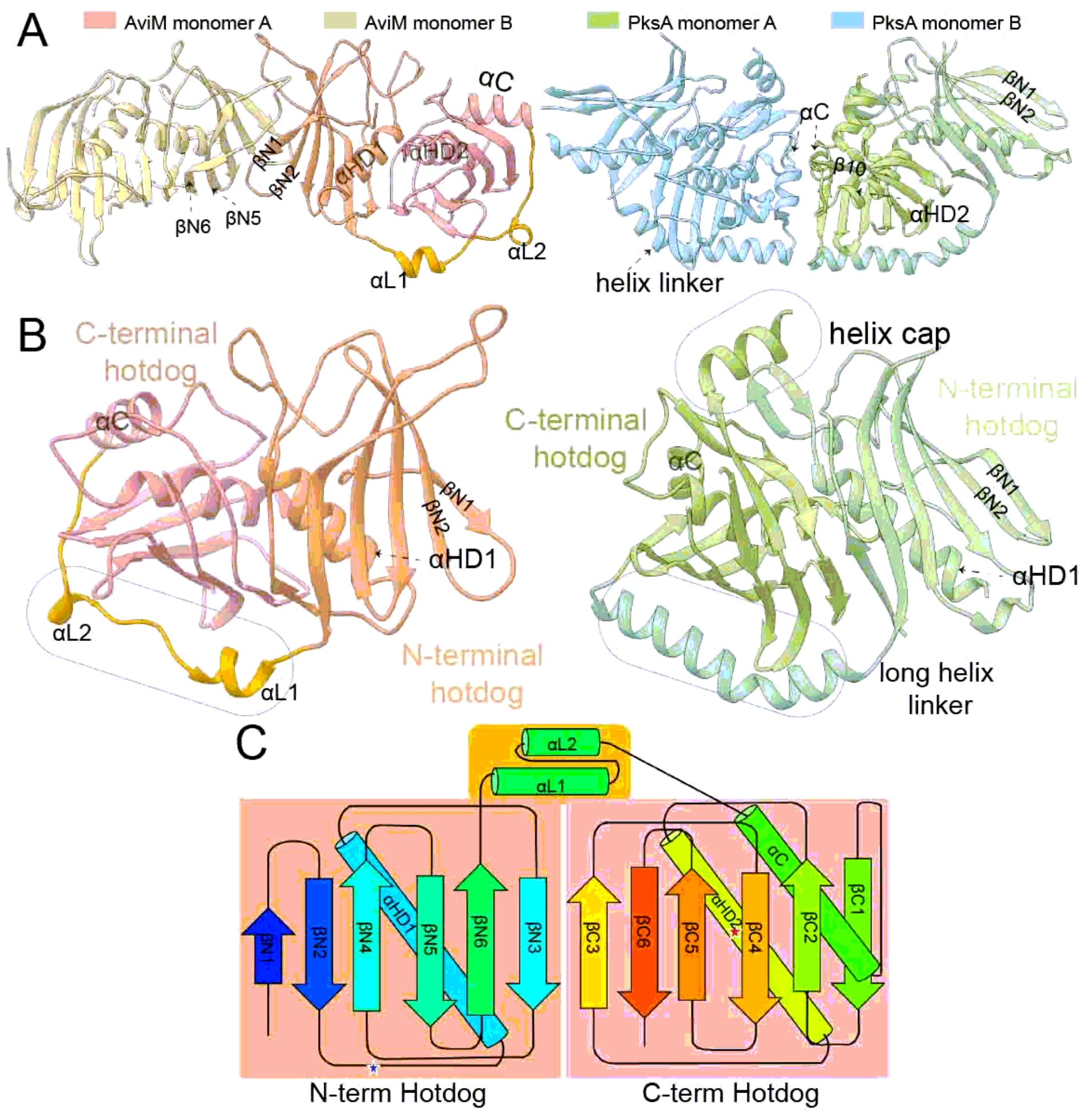

\section{Figure 3}

Overall structure of of AviM PT. and PksA PT. (A) The dimerization interface of AviM PT is different from that of PksA PT. (B) Differences between AviM PT monomer and PksA PT monomer. (C) The topology structure of AviM PT. The monomer has a N-terminal hotdog, a C-terminal hotdog and a linker containing two short a helices. 


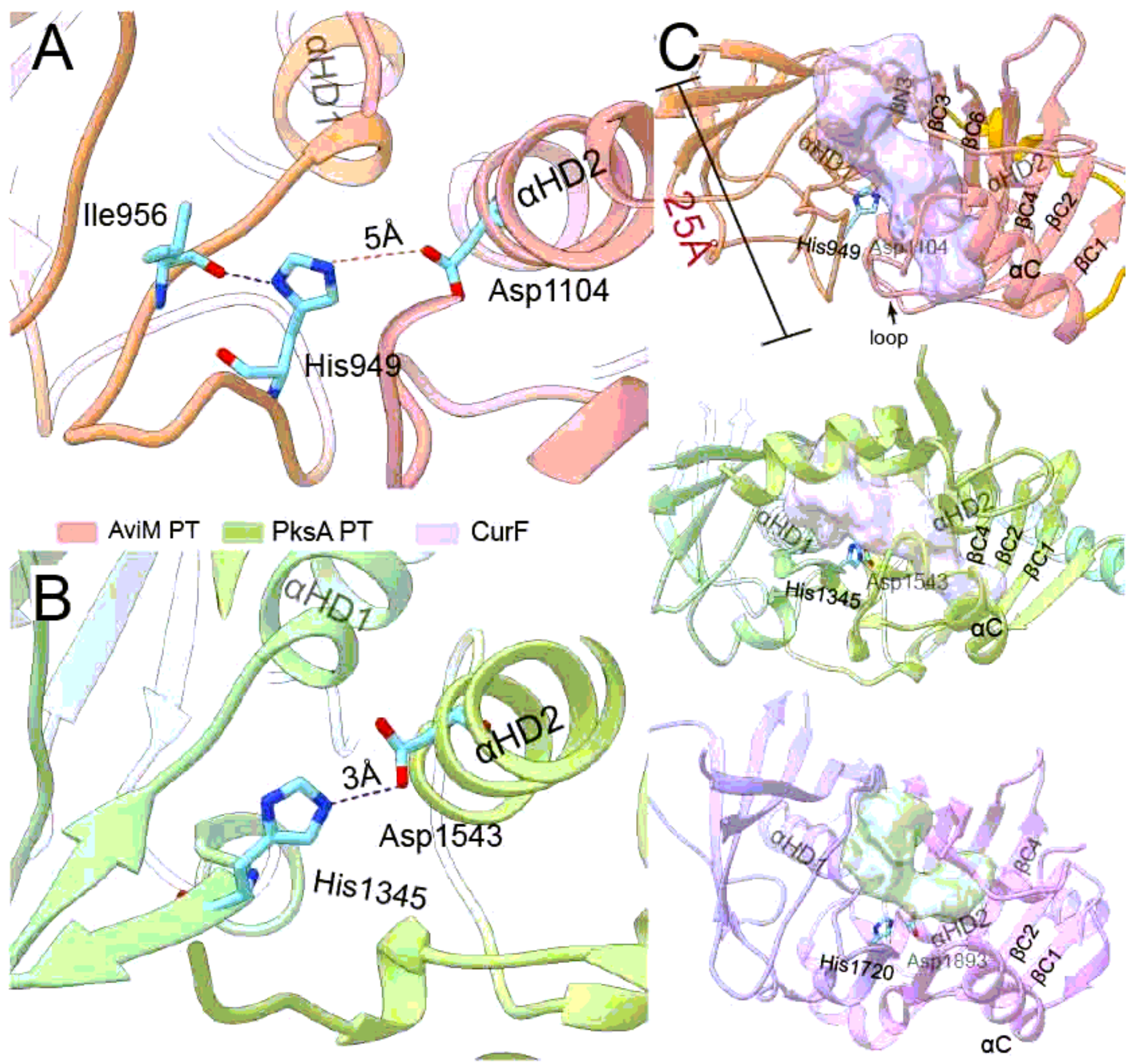

Figure 4

The active site of AviM PT. (A) The Nס of the catalytic His949 forms a hydrogen bond with the backbone carbonyl of lle956 in AviM PT. (B) The N $\varepsilon$ of the catalytic H1345 forms a hydrogen bond with the side chain of the catalytic Asp1543 in PksA PT. (C) The substrate tunnel of AviM PT is different from that of PksA PT and CurF DH. 
A
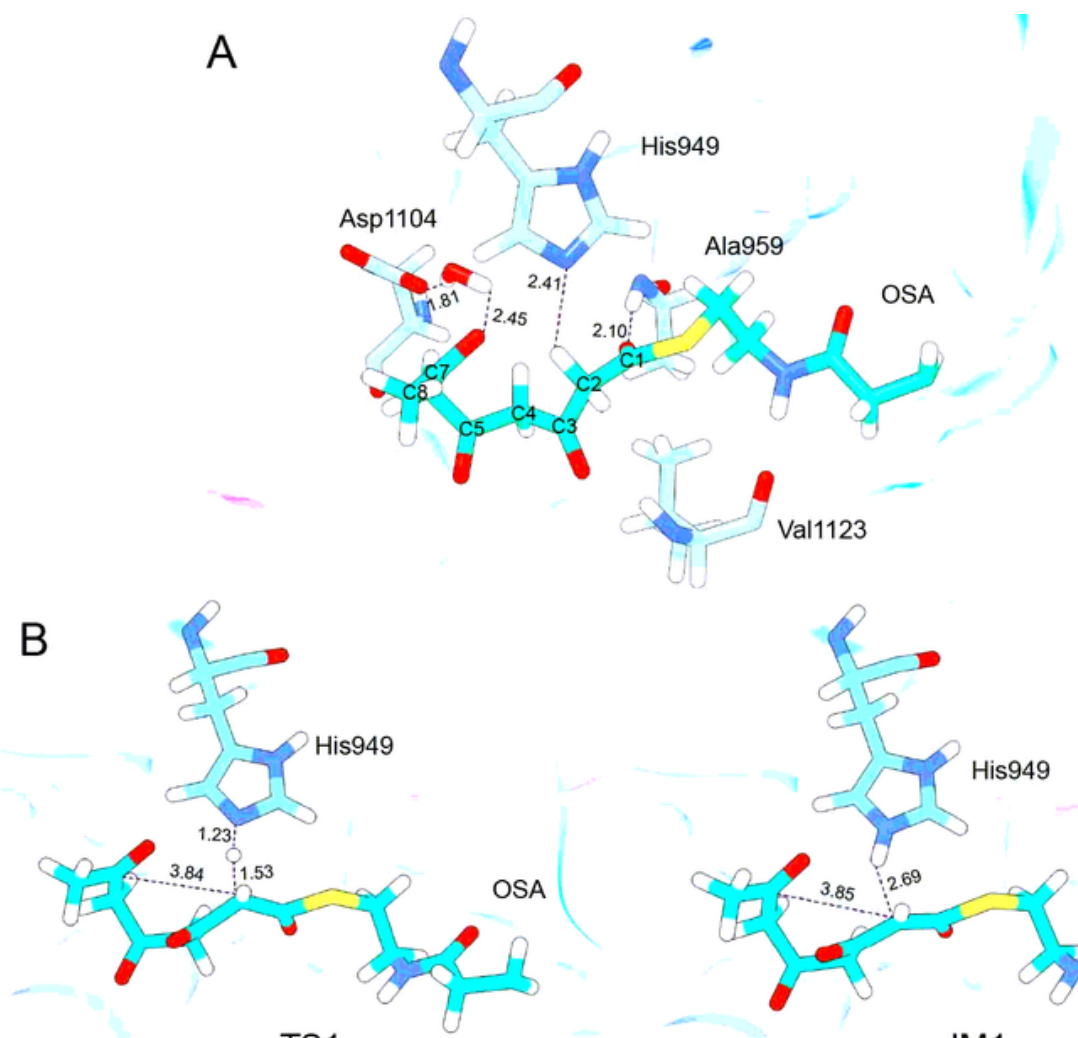

TS1

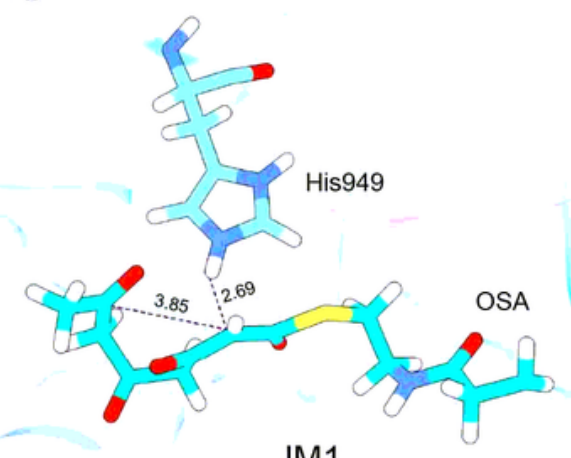

IM1

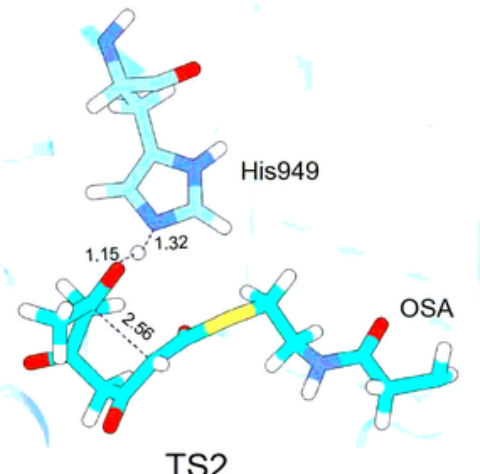

TS2

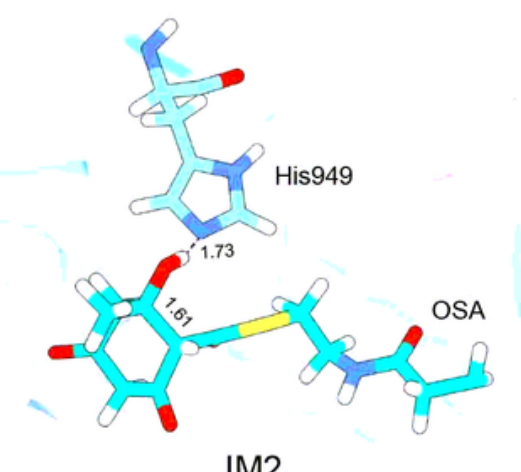

C

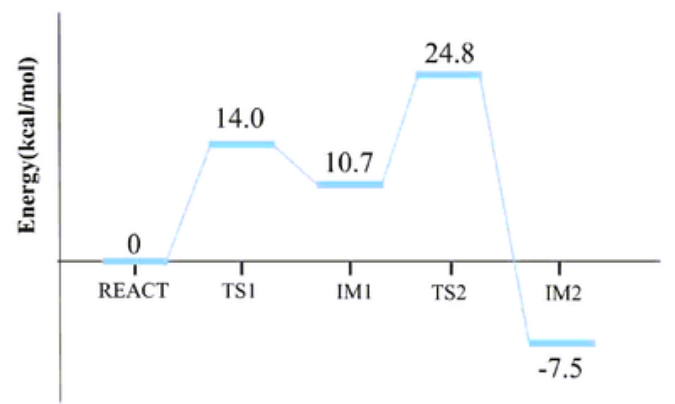

\section{Figure 5}

QM/MM calculation of AviM PT complexed with the substrate. (A) Optimized structure of reactant. (B) Structures of TS1, IM, TS2 and IM2 obtained with. (C) The energy profiles for the deprotonation of C2 and the ring formation at the M06-2x/6-31G(d): AMBER level. The distances are given in $\AA$.

\section{Supplementary Files}


This is a list of supplementary files associated with this preprint. Click to download.

- Supplementarylnformation.pdf

- Scheme1.tif 